\title{
Sensitivity of T-Wave Morphology and the QT Interval to Small Drug-Induced Electrocardiographic Changes
}

\author{
C Graff ${ }^{1}, \mathrm{~J} \mathrm{Matz}^{2}$, MP Andersen ${ }^{1}, \mathrm{JK}$ Kanters ${ }^{3,4}$, \\ E Toft ${ }^{1}$, S Pehrson $^{5}$, JJ Struijk $^{1}$ \\ ${ }^{1}$ Department of Health Science and Technology, Aalborg University, Aalborg, Denmark \\ ${ }^{2} \mathrm{H}$ Lundbeck A/S, Copenhagen, Denmark \\ ${ }^{3}$ Danish National Research Foundation Centre for Cardiac Arrhythmia (DARC), University of \\ Copenhagen, Copenhagen, Denmark \\ ${ }^{4}$ Department of Cardiology P, Gentofte University Hospital, Copenhagen, Denmark \\ ${ }^{5}$ Department of Cardiology B, Rigshospitalet, Copenhagen, Denmark
}

\begin{abstract}
The electrocardiographic $Q T$ interval is the only widely used surrogate marker of repolarization abnormality in drug trials. However, numerous basic and clinical studies have provided evidence that disturbed ventricular repolarization is reflected not only in the duration of the $Q T$ interval but also in the shape of the electrocardiographic T-wave. Thus, in order to elucidate the full spectrum of drug-induced repolarization changes, it is pertinent to perform a thorough analysis of $T$-wave morphology changes in addition to measuring the $Q T$ interval.

We present a computerized method for quantification of drug-induced T-wave morphology changes. Our results provide evidence that the shape of the $T$-wave can be a more sensitive marker of disturbed repolarization than QT interval prolongation. Effect sizes for T-wave morphology changes, $1.58 \pm 0.30$ (mean $\pm S D$ ) were 1.8 times larger than those for $Q T c F, 0.87 \pm 0.14, p=0.0013$.
\end{abstract}

\section{Introduction}

In recent years there has been a raised awareness regarding the cardiovascular safety of drugs and several drugs have been withdrawn from the market because they have been associated with the risk of developing cardiac arrhythmias [1, 2]. Most drugs that cause disturbed repolarization and arrhythmias inhibit the $\mathrm{I}_{\mathrm{Kr}}$ current and have a QT prolonging effect [3]. As a consequence, the electrocardiographic QT interval is presently used as the golden standard by which cardiac safety is assessed in "Thorough QT Studies". However, much smaller than expected QT prolongations have been reported in patients with drug-induced arrhythmias [4, 5] and important abnormalities of the repolarization sequence may not be identified by the QT interval, which characterizes only the total duration of depolarization and repolarization. Indeed, it was recently shown that abnormally shaped Twaves frequently appear without overt QT prolongation in a population of congenital LQT2 patients [6]. In addition, there are limitations to the ability to reliably detect small changes in the QT interval $(<5 \%$ of the interval) $[7,8]$.

Other ECG indices may therefore contribute importantly to assessment of drug-induced repolarization changes. Our previous work has shown that measures of $\mathrm{T}$-wave morphology can be used to discriminate between LQT1, LQT2 and healthy subjects $[9,10]$. Recognizing that a direct link has been established between inherited and drug-induced Long QT Syndrome we hypothesize that abnormal T-wave morphologies like those described in the congenital LQTS would identify small druginduced repolarization changes with a greater sensitivity than the QT interval.

\section{Methods}

\subsection{Study population and design}

Seventy-nine healthy subjects (age: $18-45 \mathrm{y}$, mean \pm SD $28 \pm 8 \mathrm{y}$ ) were included in the study. Healthy status was confirmed by history, physical examination, normal blood pressure and no use of concomitant medication. All subjects gave informed consent.

After a baseline day (day -1) where no drug was given, forty subjects (16 females, 24 males) received a single oral dose of an investigational $\mathrm{I}_{\mathrm{Kr}}$ inhibiting antipsychotic compound (Lu 35-138) on seven consecutive 
days (day 1 to day 7) while thirty-nine subjects (15 females, 24 males) received placebo.

\subsection{ECG recordings}

Three successive 12-lead resting ECGs of $10 \mathrm{~s}$ duration were recorded for each subject at $0,2,4,6$ and $12 \mathrm{~h}$ post-dose on the final study day (day 7) and at corresponding times on the baseline day (day -1 ).

\subsection{ECG processing}

Each $10 \mathrm{~s}$ ECG was used to form a median beat in the recorded leads using MUSE/Interval Editor software (GE Healthcare, Milwaukee, WI). Principal Component Analysis (PCA) was used to compute principal component median beats from the median beats in leads (I, II, V1-V6). The first principal component T-wave was used to calculate combined measure of repolarization morphology (MCS), based on asymmetry, flatness and notching. Fiducial point detection and QT measurements (Fridericia corrected, QTcF) were made automatically using the 12SL algorithm (12SL, GE Healthcare, Milwaukee, WI).

\subsection{T-wave morphology measures}

Three measures of T-wave morphology were used to identify drug-induced shape changes of the T-wave. The morphology measures were developed in earlier studies to describe the differences in T-wave asymmetry, flatness and notching between healthy subjects as representatives of normal repolarization and LQT2 subjects as a biological model of $\mathrm{I}_{\mathrm{Kr}}$ channel inhibition [11].

Asymmetry:

The difference in slope profiles of the ascending and descending parts of the T-wave was considered a measure of asymmetry. The slopes at each point of the descending part of the T-wave were mirrored to be compared with the slopes at corresponding points of the ascending segment. Both segments were normalized with the maximum of the derivative within each segment. Asymmetry was defined as the average squared difference $(d)$ between the slope segments, equation 1 .

(Eq.1) Asymmetry $\approx \frac{\sum_{n=1}^{N} d(n)^{2}}{N}$

Notch:

A curvature signal was obtained from the first and second derivatives of T-waves, equation 2 .
(Eq.2) Curvature $=-\frac{\frac{d^{2} y}{d x^{2}}}{\left[1+\left(\frac{d y}{d x}\right)^{2}\right]^{3 / 2}}$

Deflections in curvature were used to indicate the presence or absence of notches on the $\mathrm{T}$-wave. The magnitude of a notch was measured on a unit amplitude $\mathrm{T}$-wave and assigned to 1 of 3 categories as previously suggested [12]: No notch $=0$, moderate notch (perceptible bulge $)=0.5$ and pronounced notch $=1.0$ (distinct protuberance above the apex).

Flatness:

Flatness was calculated as a modified version of the standard kurtosis measure which is used statistics to describe the peakedness of a probability distribution. The $\mathrm{T}$-wave was normalized to unit area and central moments were calculated using equation 3 .

$$
M_{k}=\left[\sum_{n=1}^{N}\left(n-M_{1}\right) E C G(n)\right]^{\frac{1}{k}}, \quad M_{1}=\sum_{n=1}^{N} n E C G(n)
$$

The fourth central moment $\left(M_{4}\right)$ was normalized with the squared second moment $\left(M_{2}\right)$ and subtracted from one to let increasing values of flatness reflect increasing flatness of the T-wave, equation 4.

(Eq.4) Flatness $\approx 1-\frac{M_{4}}{M_{2}^{2}}$

Morphology Combination Score (MCS):

The three morphology measures were standardized by their relative variances and a linear combination was calculated to yield an overall description of $\mathrm{T}$-wave morphology, equation 5.

(Eq.5) $\quad$ MCS $=$ Asymmetry + Notch $+1.6 \times$ Flatness

\subsection{Effect size measures}

Effect sizes for MCS and the Fridericia corrected QT interval (QTcF) were computed on the final study day as the time matched $(0,2,4,6$ and $12 \mathrm{~h}$ post-dose) placebo corrected changes of means $(\mu)$ from baseline expressed in number of standard deviations $(\sigma)$, equation 6 .

$$
\text { Effect size }=\frac{\left(\mu_{T, p o s t}-\mu_{T, p r e}\right)-\left(\mu_{C, p o s t}-\mu_{C, p r e}\right)}{\sigma_{\text {Pooled, pre }}}
$$

where the subscripts $T$ and $C$ indicate the treatment group and the control group respectively.

Given that effect sizes are standardized, they allow direct comparison of the magnitude of change in MCS 
and QTcF to see if one measure is more sensitive to detecting disturbed repolarization than the other.

\section{Results}

Administration of the $\mathrm{I}_{\mathrm{Kr}}$ channel inhibiting investigational antipsychotic drug ( $\mathrm{Lu} \mathrm{35-138)} \mathrm{caused}$ prolongation of the mean $\mathrm{QTcF}$ and distinct morphological changes in T-wave asymmetry, flatness and the degree and presence of notches, figure 1.
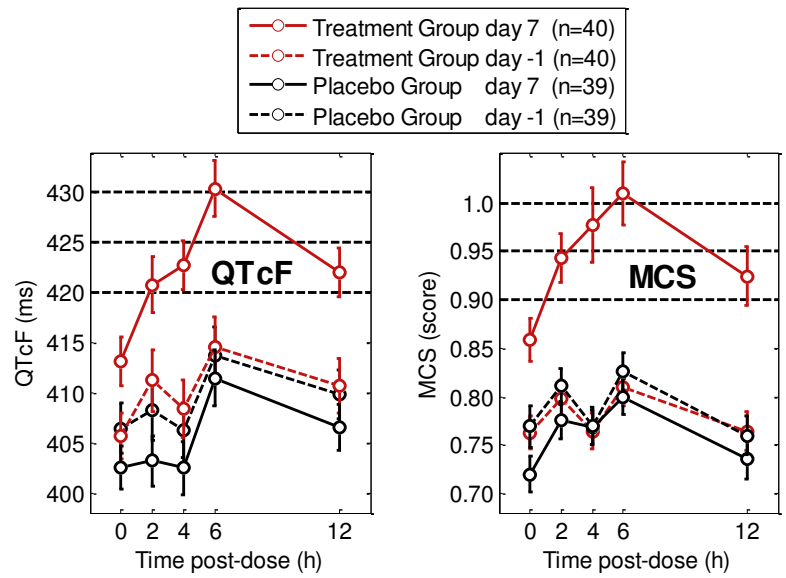

Figure 1. Treatment and placebo group at baseline and final study day. The QTcF interval and T-wave morphology measures increased after dose in the treatment group. No effect of placebo treatment was observed in the control group. I-bars indicate mean \pm 1 SE.

In general the electrocardiographic $\mathrm{T}$-waves in the treatment group were characterized by a longer duration of the ascending part of the T-wave and a noticeable decrease in the slope of that segment, leading to the appearance of asymmetric T-waves, figure 2 .

The development of a broader base and decreasing amplitude increased the flatness of T-waves. There were 6 subjects who developed notched T-waves after Lu 35138 was given, whereas no notches were identified in the placebo group.

There was a significant prolongation of the mean placebo corrected (pc: $\Delta$ treatment - $\Delta$ placebo) QTcF $\left(\mathrm{QTcF}_{\mathrm{pc}}\right)$ at study time $0 \mathrm{~h}$ on day 7, $11 \mathrm{~ms}$ [95\% CI: 8 to $13 \mathrm{~ms}], p<0.001$. At this study time a significant increase in the mean placebo corrected MCS $\left(\mathrm{MCS}_{\mathrm{pc}}\right.$ ) was also found, 0.14 [95\% CI: 0.11 to 0.17], $p<0.001$. The $\mathrm{QTcF}_{\mathrm{pc}}$ prolongations increased after dose from $11 \mathrm{~ms}(0 \mathrm{~h})$ to 18 ms $(4$ and 6 h) [95\% CI: 15 to $21 \mathrm{~ms}], p<0.001$. The $\mathrm{MCS}_{\mathrm{pc}}$ increased from $0.14(0 \mathrm{~h})$ to $0.23(6 \mathrm{~h})$ [95\% CI: 0.18 to 0.27$], p<0.001$.

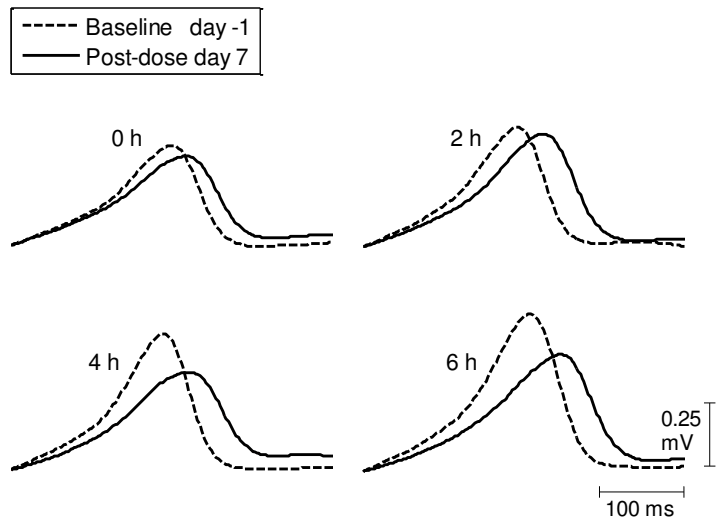

Figure 2. Time matched T-wave differences between baseline and final study day in one subject. There was an increasing prolongation of QTcF after dose and the Twave developed an increasingly distinct asymmetric and flat appearance.

The difference between effect sizes for $\mathrm{QTcF}_{\mathrm{pc}}$ and $\mathrm{MCS}_{\mathrm{pc}}$ did not reach significance before dose was given on day $7(0 \mathrm{~h}), 0.44$ [95\% CI: -0.01 to 0.89], $p=0.057$, figure 3. Post-dose, the effect sizes for $\mathrm{MCS}_{\mathrm{pc}}$ increased significantly more than the effect sizes for $\mathrm{QTcF}_{\mathrm{pc}}$, figure 3. At $6 \mathrm{~h}$ post-dose, the effect size difference between $\mathrm{MCS}_{\mathrm{pc}}$ and $\mathrm{QTcF}_{\mathrm{pc}}$ reached 0.93 [95\% CI: 0.34 to 1.53], $p<0.01$. At this study time, an additional $17 \mathrm{~ms}$ prolongation of the mean placebo corrected QTcF would have been required for $\mathrm{QTcF}_{\mathrm{pc}}$ and $\mathrm{MCS}_{\mathrm{pc}}$ effect sizes to be similar.

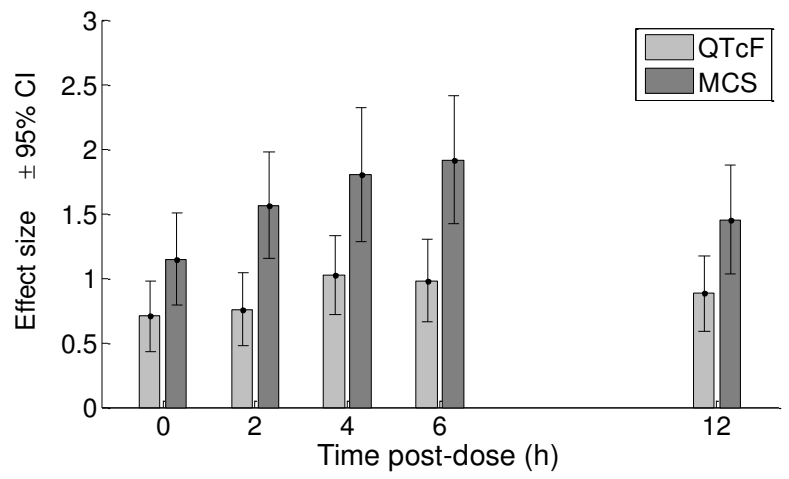

Figure 3. There was a significantly greater drug effect on T-wave morphology compared with QTcF. The time matched, placebo corrected effect sizes for MCS on day 7 were almost twice the effect sizes for QTcF.

Overall, the time matched, placebo corrected effect sizes for MCS $(1.15,1.56,1.80,1.92,1.45)$ were 1.8 times larger than those for QTcF $(0.71,0.76,1.03,0.98$, 0.88 ), $p=0.0013$, figure 3 . This difference corresponded to adding an additional $12 \mathrm{~ms}$ to the observed $\mathrm{QTcF}_{\mathrm{pc}}$ prolongation at each study time. 


\section{Discussion and conclusions}

The results of the present study suggest that it may be possible to identify drug-induced repolarization changes using computerized measures of T-wave morphology patterns that are characteristic of congenital LQT2. It was also shown that drug effects on repolarization morphology can be greater than the corresponding effect on the QT interval.

These findings could have important implications for future drug studies. First, it is widely recognized that QT interval prolongation is not the core of the problem with drugs causing arrhythmias. Most drugs are proarrhythmic because they increase inhomogeneity of repolarization. It is therefore imperative to identify electrocardiographic markers that describe cellular level inhomogeneity. Whether a drug-induced change in the morphology of the $\mathrm{T}$-wave can be used as a better surrogate marker of repolarization inhomogeneity than the QT interval, remains to be investigated. However, as a first step in the search for improved electrocardiographic markers of repolarization inhomogeneity, emphasis must be put on the sensitivity of new surrogate markers to small drug-induced repolarization changes. Our results indicate that the degree of $\mathrm{T}$-wave asymmetry, flatness and notching might be used as a more sensitive overall description of small drug-induced changes than the QT interval.

Second, most drugs that cause QT prolongation and abnormal T-waves affect the HERG channel as in congenital LQT2. Electrocardiographic expressions of abnormal repolarization in LQT2 patients would therefore be expected to be directly applicable to the identification of drug-induced repolarization changes.

We believe that computerized measures of $\mathrm{T}$-wave morphology would be an important addition to QT interval measurements because such measures may contribute to an expanded ECG safety evaluation in future drug studies through more careful characterization of repolarization abnormalities.

\section{Acknowledgements}

The authors thank H. Lundbeck A/S, (Copenhagen, Denmark) for providing the data for the present study.

\section{References}

[1] Lasser KE, Allen PD, Woolhandler SJ, Himmelstein DU, Wolfe SM, Bor DH. Timing of new black box warnings and withdrawals for prescription medications. JAMA 2002; 287: 2215-2220

[2] Roden DM. Drug induced prolongation of the QT interval. N Engl J Med 2004; 350: 1013-1022

[3] Haverkamp W, Breithart G, Camm AJ, Janse MJ, Rosen MR, Antzelevich $\mathrm{C}$, et al. The potential for QT prolongation and proarrhythmia by non-antiarrhythmic drugs: clinical and regulatory implications. Eur Heart $\mathbf{J}$ 2000; 21: 1216-1230

[4] Kay GN, Plumb VJ, Arciniegras JG, Henthorn RW, Waldo AL. Torsade de pointes: the long-short initiating sequence and other clinical features: observations in 32 patients. $\mathrm{J}$ Am Coll Cardiol 1983; 2: 806-817

[5] Bauman JL, Bauernfeind RA, Hoff JV, Strasberg B, Swiryn S, Rosen KM. Torsade de pointes due to quinidine: observations in 31 patients. Am Heart J 1984; 107: 425430

[6] Couderc JP, McNitt S, Xia J, Zareba W, Moss AJ. Repolarization morphology in adult LQT2 carriers with borderline prolonged QTc interval. Heart Rhythm 2006; 3: 1460-1466

[7] Malik M. The imprecision in heart rate correction may lead to artificial observations of drug induced QT interval changes. Pacing Clin Electro-physiol 2002; 25: 209-216

[8] Desai M, Li L, Desta Z, Malik M, Flockhart, D. Variability of heart rate correction methods for the QT interval. Br J Clin Pharmacol, 2003; 55: 511-517

[9] Kanters JK, Fanoe S, Larsen LA, Thomsen PEB, Toft E, Christiansen M. T-wave morphology analysis distinguishes between KvLQT1 and HERG mutations in Long QT Syndrome. Heart Rhythm 2004; 1: 285-292

[10] Struijk JJ, Kanters JK, Andersen MP, Hardahl T, Graff C, Christiansen $\mathrm{M}$, et al. Classification of the long-QT syndrome based on discriminant analysis of the T-wave morphology. Med Biol Eng Comput 2006; 44: 543-549

[11] Andersen MP, Xue JQ, Graff C, Hardahl TB, Toft E, Kanters JK, et al. A Robust Method for Quantification of Ikr-Related T-Wave Morphology Abnormalities. Computers in Cardiology 2007;34:341-344

[12] Lupoglazoff JM, Denjoy I, Berthet M, Neroud N, Demay $\mathrm{L}$, Richard $\mathrm{P}$, et al. Notched $\mathrm{T}$ waves on Holter recordings enhance detection of patients with LQT2 (HERG) mutations. Circulation 2001; 103: 1095-1101

Address for correspondence

Claus Graff

Department of Health Science and Technology,

Aalborg University

Fredrik Bajers Vej 7 E1-209,9220 Aalborg, Denmark

E-mail address: cgraff@hst.aau.dk 